\title{
Intermédialités
}

Histoire et théorie des arts, des lettres et des techniques

Intermediality

History and Theory of the Arts, Literature and Technologies

\section{Visage et ornement. Remarques sur une préhistoire de la visagéité photographique dans la modernité allemande chez Simmel et George}

\section{Guido Goerlitz}

Numéro 8, automne 2006

Envisager

Facing

URI : https://id.erudit.org/iderudit/1005542ar

DOI : https://doi.org/10.7202/1005542ar

Aller au sommaire du numéro

Éditeur(s)

Centre de recherche sur l'intermédialité

ISSN

1705-8546 (imprimé)

1920-3136 (numérique)

Découvrir la revue

Citer cet article

Goerlitz, G. (2006). Visage et ornement. Remarques sur une préhistoire de la visagéité photographique dans la modernité allemande chez Simmel et George. Intermédialités / Intermediality, (8), 103-119. https://doi.org/10.7202/1005542ar
Résumé de l'article

Partant de l'importance que revêt la question du visage et du gros plan dans le medium cinématographique, et de sa théorisation dans la modernité allemande de la République de Weimar chez Walter Benjamin, Bela Balazs et Siegfried Kracauer, cet article jette un regard rétrospectif sur cette question en tentant de décrire l'enracinement de la nouvelle poétique de la surface photographique dans des questions herméneutiques abordées au tournant du siècle par Georg Simmel et Stefan George. Simmel a conceptualisé la signification esthétique du visage comme un agencement centralisateur, contre le dispositif de la photographie. Le poète George donne en revanche un premier exemple d'une pratique intermédiale réunissant écriture et photographie. 


\title{
Visage et ornement. \\ Remarques sur une préhistoire de la visagéité photographique dans la modernité allemande chez Simmel et George
}

\author{
Guido Goerlitz
}

D ans l'Allemagne de la République de Weimar, après la catastrophe qu'a représentée la Première Guerre mondiale, un nombre d'intellectuels importants (notamment Béla Balázs, Siegfried Kracauer, Walter Benjamin) entreprennent d'analyser les conséquences dans la dynamique du champ social de la rupture médiologique causée par l'avènement de la photographie et de la cinématographie. Ils s'intéressent plus largement aux transformations que ces médiums entraînent par rapport à la «lecture » de la réalité ou de l'histoire. Le nouveau traitement du visage humain dans les médias techniques joue un rôle central dans les essais théoriques de ces penseurs et la surface de l'image photographique et cinématographique y est problématisée selon le paradigme de la physiognomonie, de la lecture des visages. Ainsi, Balázs se fait explicitement le défenseur d'une physionomie cinématographique et salue le cinéma comme le moment charnière d'une culture visuelle à venir, qu'il oppose au médium de l'écriture'. Dans son livre L'homme visible, qui est en même temps une des premières esthétiques du cinéma, il vise à réaliser une nouvelle sémiologie du monde visible, fondée sur les affects, inconsciente et prélogique, inaccessible au discours. Il lui prête une véritable portée anthropologique : elle aidera à créer un nouvel homme, une nouvelle communauté universelle parmi les hommes.

1. «L'invention de l'imprimerie a peu à peu rendu illisible le visage des hommes. Désormais, ils pouvaient lire tellement de papier imprimé qu'ils en sont arrivés à négliger la mimique de la communication. » Il insiste sur le fait que l'homme de cette culture visuelle engendrée par la caméra «ne remplacera pas les mots par les gestes. [...] Ses gestes signifient des concepts et des émotions qui ne pourraient absolument pas être exprimés par des mots. [...] Tout ce qui sera exprimé ici réside dans les couches profondes de l'âme.» dans Béla Balázs, L’homme visible [1923], repris dans L'esprit du cinéma, trad. Jacques M. Chavy, Paris, Payot, coll. «Bibliothèque historique», 1977, p. 42. 
Kracauer et Benjamin reconnaissent, tout comme Balázs, le potentiel utopique de la cinématographie. Toutefois, c'est en opérant un détour par l'épistémologie négative de la photographie (de son esthétique d'une surface supposée «idéologique» pour Kracauer, et de la destruction de l'aura, de l'unicité et du caractère rituel de l'image reproductible pour Benjamin) qu'ils soulignent ce potentiel émancipateur. Dans son célèbre essai, «L'œuvre d'art à l'époque de sa reproduction mécanisée », Walter Benjamin a assigné un statut particulier au thème du visage humain. Dans le cadre d'une histoire de la déchéance de l'aura dont la modernité serait profondément marquée ${ }^{2}$, il constate un dernier degré de la valeur rituelle dans la représentation du visage sur les anciennes photographies du XIX ${ }^{\mathrm{e}}$ siècle. Dans les daguerréotypes, le visage occuperait précisément une place ambivalente, à l'intersection des valeurs de la tradition et de celles des nouveaux pouvoirs du médium technique, lesquelles promeuvent ce que Benjamin appelle «la valeur d'exposition ». Le visage se situerait à la même place conceptuelle que la résistance du culte de la beauté, de la doctrine de «l'art pour l'art» qui, selon Benjamin, n'est qu'une «théologie de l'art» (OA, p. 714), contre les lignes de fuites révolutionnaires de la technique moderne.

Dans la photographie, la valeur d'exposition commence à refouler sur toute la ligne la valeur rituelle. Mais celle-ci ne cède pas le terrain sans hésiter. Elle se retient dans un ultime retranchement: la face humaine. Ce n'est point par hasard que le portrait se trouve être l'objet principal de la première photographie. Le culte du souvenir des êtres aimés, absents ou défunts, offre au sens rituel de l'œuvre d'art un dernier refuge. Dans l'expression fugitive d'un visage humain, sur d'anciennes photographies, l'aura semble jeter un dernier éclat. (OA, p. 718)

En revanche, Benjamin avait clairement dégagé la nouvelle signification qui revient aux visages dans le nouveau médium de la cinématographie : il s'extasiait devant les magnifiques «galeries physionomiques » qu'un Eisenstein aurait mises à nu. Dans l'œuvre photographique d'un August Sander (Antlitz der Zeit, 1929) qui avait présenté avec une «collection de visages » un aperçu de toutes les couches sociales de la République de Weimar, Benjamin trouvait réalisée la même tâche critique, opposée au culte du souvenir.

Dans l'essai sur «La photographie» de 1926, Kracauer situe celle-ci entre une conception métaphysique de l'écriture et de la mémoire qui relève de la mys-

2. La disparition de l'aura serait due au fait que la masse moderne « revendique que le monde lui soit rendu plus accessible» et qu’elle déprécie «l'unicité de tout phénomène en accueillant sa reproduction multiple. » (Walter Benjamin, «L'œuvre d'art à l'époque de sa reproduction mécanisée » [1936], trad. Pierre Klossowski, dans Gesammelte Schriften, Rolf Tiedemann et Hermann Schweppenhäuser (éd.), Francfort, Editions Suhrkamp, 1978, p. 713). Désormais, les références à cet ouvrage seront indiquées par le sigle «OA », suivi de la page et placées entre parenthèses dans le corps du texte. 
tique eschatologique, d'un coté, et de la cinématographie caractérisée par le procédé «révolutionnaire » du montage, de la réorganisation des morceaux de réalité représentés, de l'autre. Kracauer considère la photographie comme «la dernière étape dans la série des représentations imagées », commençant par le symbole qui traduirait «l'identité de l'homme et de la nature ${ }^{3}$. Au cours de l'histoire, l'image prendrait de plus en plus une signification dérivée, immatérielle, à mesure que grandit la domination sur la nature. La représentation symbolique devient alors de plus en plus «allégorique». Tout comme pour Benjamin, les vieilles photographies marquent chez Kracauer un seuil dans ce processus de la sémiotique des images: «Du temps des vieux daguerréotypes, la conscience est encore si impliquée dans la nature que les visages rappellent des contenus qu'il est impossible de détacher de la vie naturelle. » (P, p. 55) Par contre, «le fondement naturel vide de signification fait son apparition en même temps que la photographie». (P, p. 55) Si ainsi les «archives photographiques collectionnent les derniers éléments d'une nature aliénée au vouloir dire» (P, p. 56) elles présenteraient pourtant en même temps un pressentiment d'un nouvel ordre par «le provisoire de toutes les configurations données ». C'est finalement à la cinématographie (par le montage) que revient la possibilité utopique d'un jeu avec la nature dispersée en direction d'une «organisation valable» (P, p. 57).

Les nouvelles conceptualisations de Balázs, de Benjamin et de Kracauer possèdent néanmoins leur généalogie. Si la question de la rupture médiologique dans la modernité est associée dans leurs modèles critiques à celle d'une herméneutique du processus historique, et si le visage humain comme paradigme d’un lieu privilégié de la «lecture » devient un point névralgique dans ces théories d'une histoire de la conscience affranchie, il faut reconnaître que le réseau conceptuel qui configure les notions de visage, d'herméneutique, de sujet et de pouvoir est déjà préfiguré dans les débats esthétiques de la génération précédente.

Balázs et Kracauer sont tous les deux à leur façon des élèves du philosophe et sociologue Georg Simmel (1858-1918). Benjamin de son côté fut fortement fasciné par la poésie de Stefan George, le représentant le plus important de la poésie symboliste en Allemagne vers 1900, dont Simmel se servait comme exemple majeur pour exposer sa philosophie de l'art. Il y a donc un certain intérêt à étudier les rapports entre ce penseur des débuts de la modernité bourgeoise et la pratique esthétique dominante à son époque, c'est-à-dire celle de l'esthétisme, de

3. Siegfried Kracauer, «La photographie» [1926], dans Le voyage et la danse. Figures de ville et vues de films, textes choisis et présentés par Philippe Despoix, trad. Sabine Cornille, Paris, Presses universitaires de Vincennes, 1996, p. 54. Désormais, les références à cet article seront indiquées par le sigle « P », suivi de la page, et placées entre parenthèses dans le corps du texte. 
la poésie de «l'art pour l'art» d'un Stefan George, et de mesurer l'influence qu'ils ont pu exercer sur la génération suivante des intellectuels, qui a su théoriser de manière exemplaire l'impact des nouveaux médias. En d'autres termes: il s'agit de dégager la préhistoire, la généalogie des conceptions médiologiques avancées dans les années 1920, durant la République de Weimar, à partir d'une constellation de problèmes apparus au tournant du $\mathrm{Xx}^{\mathrm{e}}$ siècle. Car avant que le visage et la surface phénoménologique ne puissent être pensés dans le registre d’une histoire révolutionnaire et selon le modèle d'un matérialisme dialectique, il fallait d'abord penser et façonner une esthétique de la surface pure, qui se suffirait à elle-même. Ainsi, le concept d'une surface esthétiquement autonomisée (Simmel) et une nouvelle poétique intermédiale qui juxtapose la photographie et le symbolisme de l'écriture (George) ont préparé le terrain pour une «politisation » du visage et de la surface.

Pendant vingt ans, dans divers essais écrits autour de 1900, jusqu'à son grand livre sur Rembrandt4 et à «Das Problem des Porträts» (1918), le dernier article paru de son vivant, Georg Simmel avait réfléchi à la question du visage et sur les problèmes que pose la représentation de la figure humaine dans le portrait. Dans son essai «La signification esthétique du visage », paru en 1901, il théorise le visage comme une catégorie esthétique décisive dans les arts plastiques qui, en plus, s'adapterait le mieux à une économie expressive du signe visuel, étant donné que c'est le rôle même de l'esprit que «d'unifier le multiple dans les éléments du monde extérieur» et que «l'organisme représentant, dans ce sens, le premier degré de l'esprit», c'est «le visage, qui possède au plus haut point cette unité intrinsèque5. » Simmel signale comme preuve de cette unité supérieure le fait suivant: «une modification ne concernant, en réalité ou en apparence, qu'un seul élément du visage change aussitôt son caractère et son expression dans leur entier, par exemple un tremblement des lèvres, un froncement du nez, une manière de regarder, un plissement du front». (SEv, p. 137) Pour Simmel, «aucune partie ne peut être touchée par un destin quelconque sans que, comme à travers la racine commune qui les tiendrait ensemble, chacune des autres ne se trouve également atteinte par lui ». D’autre part « une unité ne prend jamais de sens et d'importance que dans la mesure où elle a en face d'elle une multiplicité dont elle constitue précisément la cohérence» (SEV, p. 138). C'est ainsi à travers cette qualité d'agencement que la «visagéité » apparaît, pour Simmel, plus qu'un

4. Georg Simmel, Rembrandt, Ein kunstphilosophischer Versuch, Leipzig, Kurt Wolff, 1916.

5. Georg Simmel, «La signification esthétique du visage» [1901], dans La tragédie de la culture et autres essais, trad. Sabine Cornille et Philippe Ivernel, Paris, Éditions Rivages, 1988, p. 137. Désormais, les références à cet ouvrage seront indiquées par le sigle «SEV », suivi de la page, et placées entre parenthèses dans le corps du texte. 
principe structurant d'organisation formelle, comme le modèle par excellence du système herméneutique, reposant sur l'idée d'interaction réciproque et d'interdépendance significative des parties et du tout. Cela dit, Simmel souligne que l'efficacité et l'intérêt esthétique de ce système sont avant tout dus au fait que «les éléments du visage sont étroitement tenus ensemble dans l'espace et ne peuvent se déplacer que dans des limites très étroites. » (SEV, p. 139) Il précise le caractère spécifique de l'unité esthétique du visage par rapport à la pluralité et la diversité de ses éléments constitutifs en soulignant l'importance du «lien avec le centre », qu'il traduit par la formule d'une «domination visible de l'esprit sur l'environnement de notre être». (SEV, p. 139) Cela implique alors en même temps toute une théorie du pouvoir et de la soumission esthétique : à l'herméneutique de la totalité du sens, même si Simmel la pense comme une «coopération» des éléments constitutifs (SEV, p. 138), correspond néanmoins l'idée politique de domination centralisée.

C'est de ce point de vue que Simmel rejette finalement la sculpture baro$q^{q u e}{ }^{6}$ : elle désavouerait «l'humanité », « tout écartement et écartèlement des parties » étant laid, « parce qu'il interrompt ou affaiblit le lien avec le centre.» (SEV, p. 139f) Le visage idéal est ainsi défini comme l'opposé exact de l'expression des figures baroques, figées dans des attitudes extrêmes et passionnées. La structure du visage prototype (dominé pour ainsi dire par un impératif catégorique) rendrait, selon Simmel,

quasi impossible pareille centrifugalité, pareille déspiritualisation. Là où elle se produit pourtant, par une bouche béante et des yeux exorbités, non seulement elle est particulièrement inesthétique, mais en outre, ces deux mouvements-là sont justement [...] l'expression d'un esprit pétrifié, d'une paralysie psychique, d'une perte momentanée de notre propre maîtrise spirituelle. (SEv, p. 139f) ${ }^{7}$

6. Le rejet du baroque signale encore à d'autres endroits de l'œuvre de Simmel un rejet de l'instant mécanique et artificiellement figé en faveur de la «modernité du mouvement», qui reste cependant par une dernière ficelle attachée à la subjectivité. L'héraclitéisme de Simmel est ainsi balancé par une structure d'organisation qu'il faudrait rapprocher de son concept de visagéité. Voir à ce sujet Lilyane Deroche-Gurcel, Simmel et la modernité, Paris, Presses universitaires de France, 1997, p. 264.

7. Sans que Simmel le dise explicitement, c'est encore tout le débat sur le groupe Laocoon et les problèmes sémiologiques qu'il a soulevés chez les auteurs allemands du XVIII ${ }^{\mathrm{e}}$ siècle (Lessing, Herder, Goethe) qui retentit dans ces formulations. La bouche entrouverte de Laocoon donnait lieu à des interprétations contradictoires: était-elle l'expression de la douleur ou au contraire montrait-elle la volonté sublime de la supprimer? Voir à ce sujet Inge Baxmann, Michael Franz, Wolfgang Schäffner (dirs.), Das Laokoon-Paradigma. Zeichenregime im 18. Jahrhundert, Berlin, Éditions Akademie, 2000. 
Or, ces déviations et ces déformations qui mettent à nu un inconscient visuel, ayant échappé au contrôle de l'esprit, sont en même temps précisément celles que le dispositif photographique a le pouvoir de dévoiler. Au sein même de sa prétention à une conception classiciste de l'expression et d'une sémiotique généralisée de la visagéité, l'esquisse théorique de Simmel témoigne ainsi des puissances déterritorialisantes et historiquement déterminées du médium photographique, contre lesquelles elle protège justement son territoire métaphysique d’âme. La conception du visage que développe Simmel constitue, en fin de compte, un geste de défense contre la technique de reproduction de la photographie qui paraît ébranler l'idée de la domination de l'esprit sur les traits du visage ${ }^{8}$.

Malgré le verdict que, parmi d'autres, le célèbre historien de l'art C. J. Burckhardt avait énoncé à la fin du XIX siècle à propos du portrait peint qui serait remplacé par la photographie ${ }^{9}$, quelques années plus tard, dans un autre essai, «Ästhetik des Porträts ${ }^{10}$ ", Simmel insiste sur la valeur d'unité esthétique que seul le travail du peintre pourrait faire ressortir. Il reprend la question du rôle du visage dans l'œuvre d'art en tant que moteur herméneutique et analyse les problèmes concrets qui découlent de sa conception du visage (comme agencement d'une surface d'éléments visibles qui sont maîtrisés par l'unité d'une âme) pour le travail artistique du peintre portraitiste.

Dans un premier temps, Simmel expose les conséquences théoriques qu’une autonomisation de la vue entraînerait. Il constate que déjà le monde extérieur, considéré comme purement visible, comme pure surface sans signification, comporte déjà dans les relations entre ses éléments des notions d'harmonie ou d'antagonisme, de généralité typique ou d'individualité, de tranquillité ou d'agitation.

8. Cette peur de perdre le contrôle de l'esprit sur le corps, dont l'esquisse de Simmel témoigne ex negativo et que le dispositif photographique a le pouvoir de révéler, est décrite de façon suggestive dans ses aspects, peu flatteurs pour le sujet, par Marcel Proust: «Mais qu'au lieu de notre œil, ce soit un objectif purement matériel, une plaque photographique, qui ait regardé, alors ce que nous verrons, par exemple dans la cour de l'Institut, au lieu de la sortie d'un académicien qui veut appeler un fiacre, ce sera sa titubation, ses précautions pour ne pas tomber en arrière, la parabole de sa chute, comme s'il était ivre ou que le sol fût couvert de verglas. » (Marcel Proust, Le côté de Guermantes [1920], dans À la recherche du temps perdu, Paris, Éditions Gallimard, coll. «Quarto», 1988, volume II, p. 439)

9. Voir Jakob Burckhardt, Die Anfänge bürgerlicher Portraitmalerei, dans Vorträge zur Kunst - und Kulturgeschichte, Rudolf Pillep (éd.), Leipzig, Éditions Sammlung Dieterich, 1987 [1918], p. 213.

10. Georg Simmel, «Ästhetik des Porträts» [1905], repris dans Aufsätze und Abhandlungen (1901-19o8), Ottstein Rammstedt (éd.), Francfort, Éditions Suhrkamp, 1993. Cet essai n'étant pas traduit en français, on suivra mon propre compte rendu et mon commentaire. 
L'impression purement sensuelle provoque ainsi déjà des sentiments qu'on aurait l'habitude d'attribuer aux choses envisagées elles-mêmes. Comme l'art du peintre n’a immédiatement à sa disposition que des «visibilités », sa signification première serait donc de rendre à la conscience lesdites relations et qualités (hylétiques, présémiotiques pour ainsi dire) qui existent entre les formes et les couleurs. Le portrait déchaîne et abstrait la visibilité de la totalité de l'homme. Selon la définition de Simmel, sa première tâche est alors de faire apparaître dans une représentation le sens de l'apparence humaine (de sa phénoménalité) - et non pas le sens derrière son apparence. Cela ne va pas de soi, souligne-t-il, pour la simple raison que cette pure surface qu'il se propose ici d'extraire artificiellement n’est pas du tout claire et évidente dans la réalité empirique. Étant donné la prédominance du psychique dans les relations entre les hommes, la représentation qu'on se fait de l'autre ne s'arrête pas à l'extérieur, l'image humaine consiste plutôt dans une confusion et une imbrication d'impressions sensuelles et psychiques. C'est à ce point de sa réflexion que Simmel prend position contre le nouveau médium de la photographie et ses bases épistémiques, c'est-à-dire contre une conception naturaliste et mécanique du monde. Si c'est le travail du portraitiste que de faire abstraction de la représentation vitale originaire (qui est hybride), de ce que nous pourrions voir de l'individu si notre œil était suffisamment indépendant et autonome, c'est pourtant le sens de l'apparence (même si c'est en tant que pure apparence) qui est en question ici : c'est-à-dire le droit, la nécessité, la signification de chaque trait extérieur dans sa relation à chaque autre, et non pas l'apparence physique et contingente telle que la photographie, selon lui, la produirait mécaniquement. La photographie est donc par cette voie exclue de manière stricte du monde du sens, qui pour Simmel ne peut être que le résultat d'un travail herméneutique de l'esprit.

Il reste cependant ce paradoxe que, en deçà des codes, des significations stables, des relations directes au sens, il y aurait au travers du travail de l'artiste une sorte de sémiotisation autre de ce domaine de l'apparence que Simmel avait eu soin au début d'isoler comme pure surface présémiotique, détachée du psychique et des significations. C'est pourquoi il est amené à mettre en lumière la différence spécifique de l'art. Dans l'art, il y aurait une nouvelle forme de nécessité qui est en opposition à la causalité naturelle. Celle-ci comprend une apparence par une autre, la déclare nécessaire en vue d'une autre, en supposant des mouvements et un échange d'énergies invisibles en dessous des surfaces (et non pas des surfaces qui s'engendreraient de manière immédiate). Dans l'art, en revanche, il existerait une telle causalité entre les surfaces. À titre d'exemple, Simmel mentionne l'arabesque dont un morceau suffit pour faire éprouver une certaine suite comme nécessaire, une autre par contre comme contingente, non issue d'une relation à l'élément visuel premier. De la même façon, il y aurait un rapport strict entre les traits d'un visage dont l'artiste par sa manière de le représenter 
nous persuaderait ${ }^{11}$. Il précise qu'il y a pour le visage une justification mutuelle des éléments de surface comme entre les incurvations ornementales dans l'arabesque, qui serait seulement plus compliquée, composée d’infiniment plus de relations. En associant ici l'ornement et le visage en vue de leur fonctionnalité esthétique, on aperçoit nettement les affinités de Simmel avec le Jugendstili2.

Si Simmel isole un terrain de l'apparence pure, il passe dans un deuxième temps à l'idée traditionnelle qu'il avait mise entre guillemets dans la première partie de sa réflexion : la prétention du portrait à représenter l'âme par le corps, avouant l'insatisfaction par rapport à l'interprétation précédente qui mettait le visage et l'arabesque sur le même plan esthétique. Sa réflexion théorique le force à admettre que des relations formelles du monde visible ne s'épuisent pas dans les simples lois de la surface. Simmel affirme que dans le portrait, l'âme possède une valeur uniquement en tant qu'âme de ce corps visible qui est montré, pas en soi. De cette façon, l'art réside dans une inversion de la relation pratique entre le corps et l'âme. L'art du portrait utilise le rapport qui, dans la vie réelle, fait de l'un le symbole de l'autre, pour arriver par son matériau à l'unité, la forme et la clarté qui seules sont sa fin. En opposition diamétrale à la pratique de la vie, l’art interprète l'extérieur de l'homme par son intérieur. Pour la production du rapport d'éléments visibles qui rend compréhensible et significatif chaque trait par rapport aux autres, l'artiste utiliserait comme le meilleur instrument et critère le fait qu'ils sont censés exprimer une âme déterminée, caractérisée d'une certaine façon. L'âme est donc le moyen pour l'induction d'une herméneutique du visible dont l'unité du visage est le paradigme.

Si les éléments visuels sont envisagés en vue de leur unité, de leurs lois et de leur organisation en tant que pures «visibilités », toutes ces relations se nouent

11. On retrouve le même paradoxe que nous avons rencontré plus haut: par la mise en forme artistique, le matériau purement visuel, non codé, regagne une force rhétorique (donc linguistique) comme le signale le terme de persuasion, utilisé par Simmel pour désigner l'esthétique de la visagéité.

12. À propos de ce rapport, David Frisby explique que «Between 1897 and 1907, Simmel frequently contributed to the Munich Jugendstil journal Jugend. Between 1900 and 1903, seven contributions consisting largely of parables appeared under the title "Momentbilder sub specie aeternitatis"-literally, snapshots viewed from the aspect of eternity. At that time, Momentbild was the word in use to describe snapshots. It still retained the literal meaning of a fleeting or momentary image or picture. But, interestingly enough, the literary "snapshots" are not accompanied by actual snapshots since Jugend was firmly committed to Jugendstil. Simmel's contributions are surrounded by Jugendstil designs, and other graphics that belong to an aesthetic movement which sought to preserve individual creativity against the reproducibility of new art forms thrown up by capitalism, such as photography. » (David Frisby, Georg Simmel, London, Chichester, 1984, p. 102) 
pourtant dans le regard du spectateur. L'âme ne peut cependant réussir à effectuer ce travail unificateur grâce à sa capacité, elle-même irréductible, à relier ses contenus dans l'unité du moi, dans la conscience de soi-même, et encore n'y parvient-elle pas arbitrairement à partir de tous les éléments. Plutôt, ceux-ci doivent offrir certaines constellations formelles et structurelles pour éveiller l'énergie unificatrice de l'âme. Ce qui apparaît comme un renforcement du caractère expressif, de la transparence des rapports et de la légitimité de leur disposition, synthétisé comme l'unité de l'apparence, n’est alors rien d'autre que la détermination à travers laquelle les puissances unificatrices de l'âme contemplative sont incitées au plus complet et au plus fort. Si la médiation à travers laquelle la mise en forme de la pure apparence se joue est en même temps l'expression sans ambiguïté de l'âme, c'est que l'unité psychique qu'elle vivifie dans le spectateur est transférée dans l'apparence. Dans la mesure où l'âme ressent sa propre activité dans une substance, elle anime cette substance elle-même, lui attribue la même unité intrinsèque et le même caractère vivant qu'elle a rappelé en elle. C'est ainsi que le cercle se clôt: l'âme, dans la mesure où elle peut être symbolisée par l'extérieur, n'est rien d'autre que le rapport unitaire des traits, les éléments du visage (et même l'œil) tiré de leur environnement, n'ayant aucune expression à eux seuls. Le travail de fond sur les visibilités, le dégagement de leur rapport nécessaire par l'artiste, conclut Simmel, n'est rien d'autre que la mise en évidence de l'âme, laquelle ne peut être pour l'artiste que le «foyer» où tous les rayons de l'apparence convergent ${ }^{13}$. À partir d'une certaine étroitesse et d'une nécessité convaincante, le rapport des traits devient alors le signe d'une âme, qui, pour ainsi dire, se cristalliserait dans ses rapports. Il s'agit là d'une sémiotique indirecte, secondaire, relationnelle ${ }^{14}$. En termes de sémiologie : le signe relationnel est en même temps

13. Cette métaphore du foyer est la trace de conceptions néoplatoniciennes qui dans la tradition allemande, à l'époque du classicisme, ont été recodifiées par K. Ph. Moritz dans le cadre de la discussion sur la question de l'ekphrasis et de la compréhension adéquate de la statuaire antique, notamment l’Apollon du Belvédère. Moritz développe, en opposition à une description dans la forme d'une narration mythopoétique telle que propose Winckelmann, un modèle généralisé et symbolique de l'idéal plastique, où les parties sont les émanations d'un point métaphysique ultérieur. Pour une analyse de cette question, voir Guido Goerlitz, Plastische Rhetorik. Allegorische und symbolische Modelle der Kunstbetrachtung bei Winckelmann und K. Ph. Moritz, dans Eckart Goebel et Achim Geisenhanslükke (dirs.), Kritik der Tradition, Festschrift für Hella Tiedemann, Würzburg, Éditions Königshausen und Neumann, 2001, p. 37-46.

14. Pour désigner le caractère « discret» du seuil sémiologique, Simmel parle encore d'un renversement d'un ordre dans un autre ordre des choses. Il le compare à une expérience scientifique où le courant électrique donne à voir un jeu de couleurs dans le tube Geissler à partir d'une certaine intensité du courant électrique. 
le plus univoque par l'intensité des relations entre les éléments, qui eux-mêmes sont encore pensés comme en dessous de la formalisation significative.

Il reste cependant une autre difficulté méthodologique : la présupposition que l'apparence d'un individu ne puisse pas seulement être portée au point de révéler sans ambiguïtés une âme, mais encore que ce soit la même âme que l'individu possède dans la réalité (psychique). Simmel résout le problème qui se pose pour le travail de l'artiste en supposant une non-correspondance physionomique entre les éléments des séries physiques et psychiques. La mise en forme ne pourrait que se poursuivre dans la direction de l'apparence, et non par une déviation ou un ajout hétérogène en faveur d'une âme qui pour l'expérience tout simplement ne sera pas symbolisée par l'apparence physique donnée. Il se tire d'affaire en arguant que l'artiste devrait développer les traits d'un visage en fonction d'une âme idéale, indépendamment de son appartenance à un homme réel ou non. L'article se termine sur une analogie avec le poème lyrique, où il faut recourir à la catégorie d'une «âme fictive» qui donne la consistance et la cohérence au produit artistique.

Déjà, dans l'essai de 1901, le parallélisme entre système herméneutique, régime significatif et subjectivation (chrétienne) était associé aux problèmes reliés à l'esthétique de la "poésie pure», qui pour ainsi dire est soumise aux mêmes exigences éthiques d'un self-control que le «visage catégorique » postulé là. Or, Simmel fut un des premiers à établir, auprès d'un public élargi, la réputation du poète Stefan George (1868-1933), qui fut le représentant le plus important de l'esthétisme et d'une poésie artiste, sophistiquée et autoréflexive en Allemagne. George est, comme le signale Jean-Louis Vieillard-Baron, commentateur des écrits esthétiques de Simmel, «un poète méconnu en France, sauf par l'élève de Simmel que fut Charles le Bos ${ }^{15}$. » Habitué des fameux «mardis » de Mallarmé dans les années 1890 à Paris, Stefan George fut un traducteur éminent de Baudelaire et introduisit l'esthétique du symbolisme français en Allemagne. Sa propre poésie est caractérisée par une langue hiératique et dure, par un travail rigoureux sur le matériau linguistique. Même ses adversaires devaient reconnaître qu'il avait élevé le langage poétique, surtout sur le plan phonétique et musical, à un nouveau degré de perfection. Il est mort à Locarno, en exil volontaire, après l'accession de Hitler au pouvoir. Cependant, à cause de son antidémocratisme déclaré et de ses tentatives d'exercer à partir de son esthétisme un pouvoir charismatique, de former avec son Cercle un état d'élite au cœur même de la société, il fut rapproché plus tard, notamment par les penseurs de la théorie critique,

15. Georg Simmel, «Stefan George. Une recherche de philosophie de l'art» [1901], dans Philosophie de la modernité, introd. et trad. Jean-Louis Vieillard-Baron, Paris, Éditions Payot, 1990, p. 129 (note du traducteur). 
sinon aux tendances préfascistes, du moins à la déchéance des valeurs politiques de l'ère bourgeoise. Dans son essai «Stefan George. Une recherche de philosophie d'art», Simmel expose, bien qu'il ne parle pas explicitement du visage, des réflexions de métaphysique artistique qui, par leurs analogies frappantes avec ses analyses sur le visage, suggèrent pourtant assez clairement un réseau conceptuel commun $^{16}$. En rupture avec l'épistémè naturaliste qui traverse toute la poésie lyrique du XIX ${ }^{\mathrm{e}}$ siècle (la Erlebnisdichtung), chez George, le contenu serait conçu comme un moyen pur et simple de constituer des valeurs purement esthétiques. Simmel donne alors une définition de la poésie «artistique» qui cite presque littéralement celle sur le caractère des traits de visages: «Plus la logique interne de l'œuvre d'art est serrée, plus cette unité intime se manifeste dans le fait que toute altération, même la plus légère de ce qu'on appelle forme est aussitôt une altération du tout, est donc aussi de ce qu'on appelle contenu, et inversement ${ }^{17}$. » De plus, l'argument sur le processus projectif dans la production d'une unité esthétique par le spectateur-lecteur est identique: la personnalité créatrice à laquelle l'œuvre d'art est attribuée ne serait que la condition intrinsèque de notre propre perception, une fonction de l'œuvre d'art donnée elle-même, elle n'est pas une cause extérieure comme la personnalité historique, mais une loi immanente de cohérence. Il est d'ailleurs significatif que, selon Simmel, c'est le thème de la résignation érotique ou de la domination des affects dans la poésie de George qui incarnerait le mieux, sur le plan du contenu, le moment de distance par rapport à la «vie naturaliste » dont témoignerait essentiellement sa composition artistique rigide sur le plan de la forme. Laccent qu'il met sur la maîtrise de soi de la part de George s'accorde bien avec l'horreur que Simmel éprouve devant l'attitude passionnée de la plastique baroque, aussi bien que devant les moments non contrôlés, contingents, vidés de sens, que la photographie, selon lui, sait tirer de la réalité.

La poésie de George est, comme il a été souligné avec insistance dans la recherche récente ${ }^{18}$, profondément marquée par son caractère rituel. On a ainsi relevé la signification des éléments eucharistiques et catholiques dans la politique

16. C’est le genre lyrique lui-même qui acquiert ici le statut de visagéité par sa densité et son organisation rigoureuse: "S'il est vrai que l'essence de l'âme est l'unité du divers - tandis que toute corporéité est condamnée à une insurmontable extériorité aucune forme d'art sinon la poésie lyrique, en raison de sa petite dimension qu'on peut embrasser d'un seul regard, n'est plus apte à faire ressentir et à faire agir cette force et ce secret de l'âme. » (Georg Simmel, «Stefan George», p. 129)

17. Georg Simmel, «Stefan George», p. 132.

18. Voir Wolfgang Baumgart, Ästhetischer Katholizismus, Stefan Georges Rituale der Literatur, Tübingen, Editions Niemeyer, 1997 et Rainer Kolk, Literarische Gruppenbildung am Beispiel des George-Kreises (1895-1945), Tübingen, Éditions Niemeyer, 1998. 
culturelle et la pédagogie de son cercle éminent de poètes et de savants, qui visait à une restauration nationale par le travail poétique d'une restauration de la langue. Adorno avait déjà associé auparavant cet aspect de la figure de George au phénomène du narcissisme ${ }^{19}$.

George a bel et bien pratiqué, de diverses manières, le geste d'ésotérisme: d'abord celui de l'exigence esthétique, excluant, selon ses propres termes, celui qui ne pouvait ou ne voulait pas concevoir l'œuvre poétique comme une création formelle; plus tard celui d'un cercle de restauration culturelle et politique, regroupé de manière informelle autour de sa personne, incarnant soi-disant une Allemagne secrète. [...] C'est précisément le ton ésotérique, ce narcissisme distant, qui, selon Freud, permet aux personnages de chefs politiques d'exercer un effet psychologique sur la masse, qui y contribua [...] La langue anglaise dispose pour cela d'une expression intraduisible et parfaite: self-styled ${ }^{2 \circ}$.

Benjamin aurait, selon Adorno, sans doute été «le premier à rattacher l'œuvre poétique de George au Jugendstil, si manifeste dans l'ornementation typographique de Lechter [l'illustrateur des livres de George et du Cercle]. » (AG, p. 381) À partir de ce rapprochement, la poésie lyrique de George devrait être conçue comme celle d'un «ornement inventé, d'une impossibilité. » (AG, p. 381) Dans la nécessité de l'inventer par contre, elle serait plus que simplement ornementale, «elle est l'expression d'un besoin aussi critique que désespéré » (AG, p. 381). En effet, les stratégies de George dans la publication de ses œuvres réunissaient l'art et l'artisanat. Il faut y voir des stratégies qui rendaient possible de publier pour la masse et de conserver en même temps une exclusivité. George maniait les éditions publiques de façon à faire référence aux éditions de luxe qui les précédaient. Ainsi il arrivait à faire apparaître celles-ci comme un remplacement acceptable des précieuses éditions privées. Mais il y a plus. Selon Adorno, même

[...] la vie de communauté souhaitée par George a une coloration décorative [...]. Avec les choses, les individus sont eux aussi victimes du déclin vers les arts décoratifs: la décoration est le stigmate de la beauté émancipée. Elle succombe dès que les nouveaux sujets, techniquement maîtrisés, peuvent être produits à volonté, à bon marché et deviennent commercialisables. George n'est pas loin d'en être conscient dans le poème final des Pilgerfahrten (Pèlerinages, 1891) qui fait la transition vers

19. Cette lecture rejoint les propos de Simmel sur la fonction de la «résignation» pour le régime de signes mis en œuvre dans sa poésie. Simmel avait aussi forgé la formule clé d'une «intimité monumentale » pour la désigner.

20. Theodor W. Adorno, «George» [1967], dans Notes sur la littérature, Paris, Éditions Flammarion, 1984, p. 374. Désormais, les références à cet article seront indiquées par les sigle «AG», suivi de la page, et placées entre parenthèses dans le corps du texte. 
Algabal (1892)21. Il évoque l'idéal de la beauté par l'image de l'agrafe: «Je la voulais en froid métal / Semblable à une languette lisse et ferme, / Mais dans les puits, sur tous les rails, / Aucun métal n'était mûr pour la fonte. / Maintenant cependant je la veux ainsi: / Telle une étrange et grande ombrelle, / Formé d'or rouge feu / Et de riches pierreries étincelantes.» $\mathrm{Si}$ «aucun métal n’était mûr pour la fonte», les conditions de la vie matérielle refusant la possibilité objective du beau qui s'ouvre au contraire, telle «une étrange et grande ombrelle», surgissant de façon chimérique dans la négation de la vie matérielle, alors celle-ci absorbe à son tour la chimère par l'imitation. La simple agrafe produite par les arts décoratifs en fer à bon marché représentait de façon allégorique l'agrafe qu'il fallait couler en or parce que le fer véritable faisait défaut. La correspondance ne laisse aucun doute sur le caractère chimérique des choses exquises. Elles sont le produit de machinations économiques ${ }^{22}$.

Comme exemple de cette disposition, Adorno rappelle que le «pathos bibliophile» de George avait inventé des caractères d'imprimerie qui imitent sa propre écriture (une écriture sans majuscules qui était aussi pratiquée comme un signe de reconnaissance par les membres du Cercle). Il y voit «le mensonge Art déco » d'un produit de la technique de masse qui se présente comme une chose originelle et qui « est dû à la nécessité d’une mise en scène qui ne dispose d'aucun critère objectif du beau mais seulement du maigre programme : d'innover. » (GH, p. 223) Le beau symboliste de George serait doublement déformé: par une croyance au matériau, et par une ubiquité allégorique. «Sur le marché Art déco », conclut Adorno, « tout peut tout signifier. » (GH, p. 223)

Il est cependant raisonnable de rattacher également le poème sur l'agrafe à la question des pratiques médiatiques, car on peut y lire à travers la concurrence des matériaux une autre concurrence, liée aux modes de représentation. Si la première agrafe est en dehors de sa substantialité ferme désignée par l'attribut de la froideur, la seconde contient une qualification de couleur («rouge feu») et de lumière («étincelantes»). Or, l'association de la matière de l'or (comme le référent stable reterritorialisant du système monétaire) et de la nature chimérique de «l'étrange ombrelle» dans la seconde, combine justement les aspects ambivalents de la photographie: sa référentialité manifeste et son caractère à la fois contingent, incertain, fantomatique de «non-signe», de «non-langage» (Barthes).

21. C'est-à-dire l'empereur de la Rome décadente dont le royaume artificiel était dans la poésie de George le chiffre de l'art pour l'art.

22. Theodor W. Adorno, «George et Hofmannsthal. À propos de leur correspondance (1891-1906)» [1940], dans Prismes, trad. Geneviève Rochlitz et Rainer Rochlitz, Paris, Payot, 2003, p. 222. Désormais, les références à cet ouvrage seront indiquées par le sigle «GH », suivi de la page et placées entre parenthèses dans le corps du texte. 
On peut finalement dégager les traces d'une telle constellation qui chancelle entre la substantialité de l'écriture symbolique et la représentation photographique dans la pratique poétique même de George. Celle-ci implique également le calcul stratégique du paratexte et de l'aspect visuel du livre. Cela dit, parmi les moyens employés par le poète pour exercer un véritable pouvoir charismatique, l'image faciale possède un statut éminent. Nombreux sont les exemples où le visage de George acquiert aux yeux de ses disciples une fonction cathartique qui (en accord avec ce que Simmel dit sur la forme de la résignation) sert de remède thérapeutique en domptant des forces «dionysiaques »23. Elle donne un centre à la vie des adeptes. La domination symbolique que George a exercée en premier lieu dans son Cénacle est fondue dans son apparence physique et sa présence physionomique même en son absence, soit dans l'image (reproduite, photographiquement). Ainsi, déjà de son vivant, les photographies de George étaient célèbres et largement répandues.

Si Simmel avait conçu la poésie artistique symboliste de George en parallèle avec le modèle d'un visage catégorique, centralisateur et dominateur, il avait pourtant strictement exclu la surface photographique du domaine de ce travail esthétique et éthique. George s'est par contre lui-même largement servi du nouveau médium technique afin de produire des « effets de visagéité » dans le public. Dans une lettre de $1912^{24}$, Friedrich Gundolf, le plus proche de ses élèves, réclame la mise à disposition des photos du maître en recourant à l'argument que depuis longtemps sa tête serait devenu une affaire publique, et que c'était l'effet cathartique et la conception de la réalité du poète qu'elle dégagerait qui devraient être propagés en vue d'une reconnaissance générale. En effet, déjà en 1904, George avait inséré dans les Blätter für die Kunst, le principal organe de publication du Cénacle, un collage (fig. 1) réunissant les photos de poètes amis, proches de

23. C'est ainsi que dans une lettre du 4 décembre 1898 , Rilke demande à un ami la reproduction photographique d'un portrait de George en expliquant: «Stephan Georges Verse vollenden sich ja in ihm. Man muß das weise Schweigen dieser Lippen schauen, um die Rhythmen ihrer Beredtsamkeit zu begreifen. [Les vers de Stephan George s'y accomplissent. Il faut voir le sage silence de ces lèvres pour comprendre les rythmes de leur éloquence.] » (Je traduis) Pour Rilke, le visage certifie et authentifie l'œuvre; il est le lieu même où la personne et l'œuvre s'unissent. Le poète George a été sensiblement attentif à tous ces aspects rituels de la communication artistique. Aucun auteur de la modernité allemande n'a occupé et contrôlé avec une pareille conscience les zones marginales de son œuvre (tout ce qui, selon la terminologie de Genette, relève du «paratexte»). Voir Wolfgang Braungart, Ästhetischer Katholizismus, Stefan Georges Rituale der Literatur, Tübingen, Éditions Niemeyer, 1997, p. 124f.

24. Stefan George - Friedrich Gundolf, Briefwechsel, Munich, Éditions Küpper, 1962 , p. 236. 


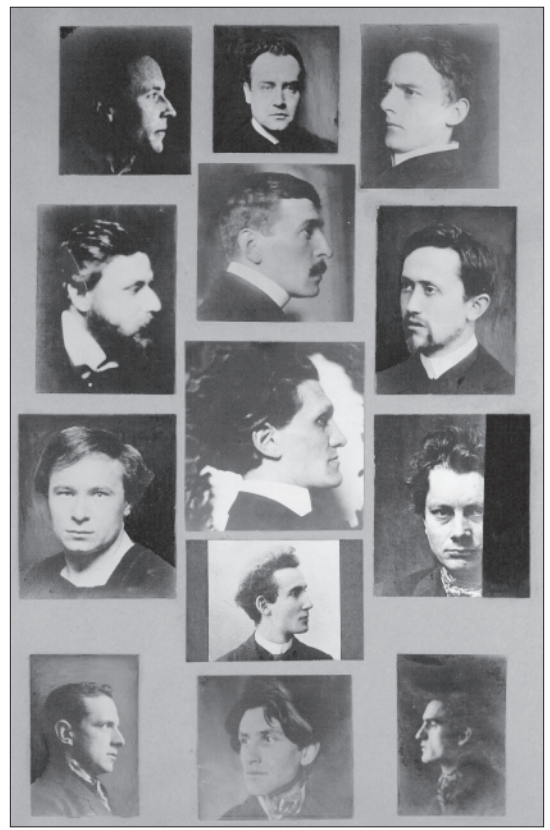

Fig. 1. Dichtertafel, Blätter für die Kunst, 1904. Avec l'aimable autorisation de l'archive Stefan George, Württembergische Landesbibliothek Stuttgart.

son programme artistique, centrées autour de la sienne (ce qui est absolument extraordinaire à cette époque-là, les collages n'étant devenus une technique privilégiée par les cubistes que bien après). Or, ce Dichtertafel (tableau de poètes) se rapportait explicitement à une collection de vers (dans le cycle lyrique Lannée de l'âme, 1897) concernant justement le souvenir des représentés. Elle portait comme titre : "VERSTATTET DIES SPIEL: EUERE FLÜCHTIG GESCHNITTENEN SCHATTEN ZUM SCHMUCK FÜR MEINER ANDENKEN $\mathrm{SAAL}^{25}$ ». Photographie et écriture sont alors effectivement placées au même rang, traitées comme des équivalents. Enfin, après la mort d'un jeune membre du Cercle, Maximilian Kronberger, à l'âge de 17 ans, George initia un culte l'élevant à la position d'un dieu, «Maximin». Sur le frontispice d'un livre de poésies en souvenir du défunt (Maximin - Ein Gedenkbuch, 1907), George fait figurer une photographie du poète adolescent qui est encadrée par un ornement

25. «Permettez ce jeu: vos ombres fugitivement coupées comme ornement pour la salle des mes souvenirs.» (Je traduis) 


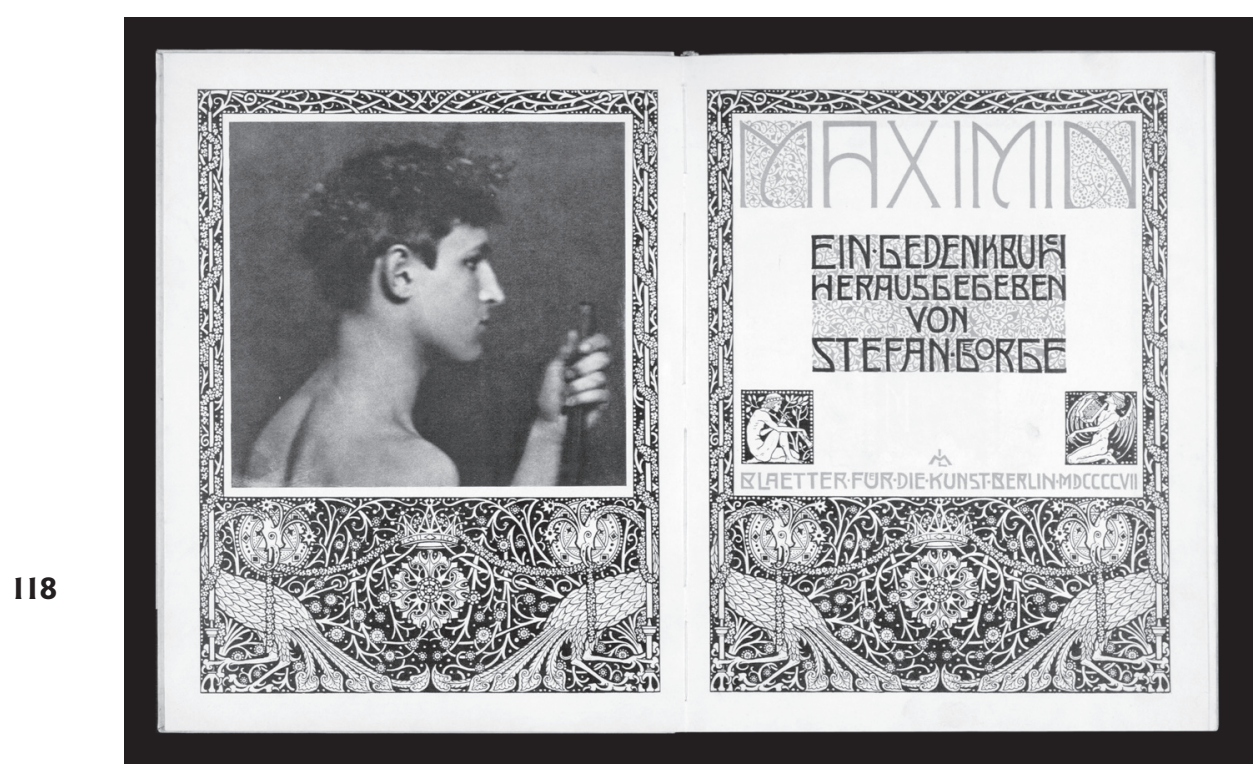

Fig. 2. Frontispiz, Gedenkbuch für Maximin, 1907. Avec l'aimable autorisation de l'archive Stefan George, Württembergische Landesbibliothek Stuttgart.

de riches arabesques réalisé par Melchior Lechter (fig. 2). Cornelia Blasberg a raison quand elle souligne que ce n'est pas Maximin, le dieu sacralisé par le médium de l'écriture qu'on voit sur la photo, mais bien Maximilian, le mortel, un garçon ordinaire ${ }^{26}$. Simmel avait comparé l'arabesque et la formalisation de la «facialité» dans le portrait peint par opposition à la photo. Ici, dans la photo du frontispice, il est cependant significatif de constater qu'on n'a pas une vue frontale du visage - la tête est détournée vers le fond de l'image à droite, dans la direction du cadre ornemental, dans un lieu invisible où la vie se transforme en art. En même temps qu'il reprend le topos de la «nécessité» du visage par la mise en scène quasi sculpturale du profil, George semble néanmoins retenir au sein de l'art, à travers la sémiologie de la photographie, la différence entre l'art autonome et la mortalité en tant que contingence de l'instantané.

26. Cornelia Blasberg, «Stefan Georges Jahr der Seele. Poetik zwischen Schrift und Bild», dans Hofmannstahl-Jahrbuch, Freiburg, Éditions Rombach, 1997, p. 236. 
C'est encore Adorno qui avait souligné cette description de rêve dans le seul recueil de prose laissée par le poète George, un véritable cauchemar :

\section{La tête qui parle}

On m’avait donné un masque d'argile qu'on avait accroché au mur de ma chambre. J'invitai mes amis afin qu'ils me voient faire parler cette tête. Je lui ordonnai distinctement de dire le nom de celui que je désignerais et comme elle restait muette je tentais d'écarter ses lèvres avec mon doigt. Alors elle grimaça et me mordit le doigt. Je répétai l'ordre à haute voix et avec une volonté extrêmement tendue en désignant quelqu'un d'autre. Alors elle prononça ce nom. Nous quittâmes tous la pièce saisis d'horreur et je sus que je n'y reviendrais jamais. (AG, p. 383)

Adorno commente en ces termes: «La violence obligeant encore une fois à prononcer le nom, son triomphe et l'horreur infinie qu'il provoque parce qu'il se détruit lui-même - voilà l'énigme de George.» (AG, p. 383) Si l'idéologie de «l'art pour l'art» a amené George à une herméneutique stricte de la surface esthétique, d'un matériau linguistique affiné et lissé, à un «régime de signes signifiants » allégorique où «tout peut tout signifier », le poète indique pourtant le prix de cette entreprise (le pouvoir charismatique auquel précède une mise à mort, la forme de la résignation, dont la photographie, «fonction du temps qui s'écoule» (P, p. 48), est le reflet), manifeste dans sa pratique intermédiale et son appui à la poétique de la photographie à laquelle il confronte le symbolisme de l'écriture.

En suivant le fil conducteur d'une herméneutique du visage et de la surface phénoménale dans l'œuvre d'art, notre discussion de Simmel et de George nous a permis de voir dans quelle mesure l'idéologie «bourgeoise » de la stabilité du sujet s'est engagée dans une constellation paradoxale, révélée par les problèmes que pose sa représentation dans les médias techniques. Il nous est alors permis de comprendre pourquoi la génération suivante devait dynamiser la question du visage. La totalité du sens qu'on lui avait imputée comme une substance inaliénable paraissait désormais, après la catastrophe de la Grande Guerre, après l'expérience historique d'une destruction et d'une fragmentation inouïe, obsolète. 